\title{
Correction: Sarcopenia and sarcopenic obesity are independent adverse prognostic factors in resectable pancreatic ductal adenocarcinoma
}

\author{
Elisabeth S. Gruber, Gerd Jomrich, Dietmar TamandI, Michael Gnant, Martin Schindl, \\ Klaus Sahora
}

There are errors in the author affiliations. The correct affiliations are as follows:

Elisabeth S. Gruber ${ }^{1.3}$, Gerd Jomrich ${ }^{1,3}$, Dietmar Tamandl ${ }^{2}$, Michael Gnant ${ }^{3}$, Martin Schindl ${ }^{1,3}$, Klaus Sahora ${ }^{1,3}$

1 Division of General Surgery, Department of Surgery, Medical University of Vienna, Vienna, Austria, 2 Department of Biomedical Imaging and Image-guided Therapy, Medical University of Vienna, Vienna, Austria, 3 Comprehensive Cancer Center, Medical University of Vienna, Vienna, Austria.

\section{Reference}

1. Gruber ES, Jomrich G, TamandI D, Gnant M, Schindl M, Sahora K (2019) Sarcopenia and sarcopenic obesity are independent adverse prognostic factors in resectable pancreatic ductal adenocarcinoma. PLoS ONE 14(5): e0215915. https://doi.org/10.1371/journal.pone.0215915 PMID: 31059520

\section{f openaccess}

Citation: Gruber ES, Jomrich G, Tamandl D, Gnant M, Schindl M, Sahora K (2020) Correction: Sarcopenia and sarcopenic obesity are independent adverse prognostic factors in resectable pancreatic ductal adenocarcinoma. PLoS ONE 15(12): e0244896. https://doi.org/ 10.1371/journal.pone.0244896

Published: December 29, 2020

Copyright: @ 2020 Gruber et al. This is an open access article distributed under the terms of the Creative Commons Attribution License, which permits unrestricted use, distribution, and reproduction in any medium, provided the original author and source are credited. 